\title{
Interference detection method using wireless LAN based MIMO transmission
}

\author{
Ryochi Kataoka $^{1 \mathrm{a})}$, Kentaro Nishimori ${ }^{1}$, Masaaki Kawahara ${ }^{1}$, \\ Takefumi Hiraguri ${ }^{2}$, and Hideo Makino ${ }^{1}$ \\ ${ }^{1}$ Graduate School of Science and Technology, Niigata University \\ 8050, Ikarashi 2-no-cho, Nishi-ku, Niigata, 950-2181, Japan \\ ${ }^{2}$ Nippon Institute of Technology \\ 4-1 Gakuendai, Miyashiro-machi, Minamisaitama-gun, Saitama Pref. 345-8501, \\ Japan \\ a)kataoka@gis.ie.niigata-u.ac.jp
}

\begin{abstract}
This paper proposes an interference detection method in MIMO transmission, which utilizes periodical preamble signals in a frequency domain. In the propose method, second antenna receives the signals while first antenna transmits the preamble signals. Hence, the interference can be detected by observing the subcarriers which are not mapped short preamble signal using the received signal after the FFT processing. Moreover, we utilize the orthogonal polarized antennas to reduce the mutual coupling between the transmitter and receiver. By a computer simulation and measurement with the use of orthogonal polarized antenna, it is shown that the proposed method can successfully detect interference from the other system when the interfering power is greater than the noise power.
\end{abstract}

Keywords: MIMO, collision detection, short preamble signals, orthogonal polarized antennas, interference

Classification: Antennas and Propagation

\section{References}

[1] IEEE 802.11 Standard for Local and Metropolitan Area Networks Part 11: Wireless LAN Medium Access Control (MAC) and Physical Layer (PHY) Specifications, March 2012.

[2] IEEE 802.3 Standard for Information technology-Specific requirements - Part 3: Carrier Sense Multiple Access with Collision Detection (CSMA/CD) Access Method and Physical Layer Specifications, 2008.

[3] T. Hiraguri, K. Nishimori, T. Ogawa, R. Kataoka, H. Takase, and H. Hideo, "Access control scheme for collision detection utilizing MIMO transmission," IEICE Commun. Express, vol. 2, no. 4, pp. 129-134, April 2013.

[4] A. Paulaj, R. Nabar, and D. Gore, "Introduction to space-time wireless communications," Cambridge University Press 2003.

[5] K. Nishimori, K. Ushiki, and N. Honma, "Experimental Evaluation Toward Transmit and Receive Diversity Effect in SIMO/MIMO Sensors," Proc. EuCAP2012, POST2.9, April 2012. 


\section{Introduction}

Recently, wireless LAN (WLAN) devices are equipped in many user terminals (UT). Therefore, access points (APs) of WLAN are widely introduced at offices and houses. The interference can be reduced to some extent thanks to the carrier sense [1]. However, the collision occurs between the WLAN devices with IEEE $802.11 \mathrm{~b} / \mathrm{g} / \mathrm{n}$ standard and products such as microwave oven at $2.4 \mathrm{GHz}$ band. In wired LAN, an access control scheme called carrier sense multiple access/collision detection (CSMA/CD) is introduced [2]. The wired LAN can detect the packet collision by the voltage variation inside the Ethernet cable before packet transmission. Since the wired LAN employs packet contention detection in advance and re-transmission immediately, transmission efficiency is over $90 \%$ [3]. On the other hand, the access control method called CSMA/collision avoidance (CSMA/CA) is adopted in WLAN [1]. Unlike wired LAN, it is difficult to detect the packet collision when the signals are transmitted. Hence, the reception characteristic is judged by the reply of acknowledgement (ACK) by a receiving station. Compared to the wired LAN, the transmission efficiency of WLAN is very small and its value is less than $65 \%$, because the re-transmission cannot be employed when the collision occurs [3]. Hence, the interference detection when the transmission is employed is one of key issues for high efficient communication.

This letter proposes a interference detection method using multiple-input multiple-output (MIMO) transmission [4]. The proposed method utilizes the fact that the second antenna is idle when the first antenna transmit the short preamble signals which are used for timing synchronization with the UT. The signals are mapped in only several subcarriers in short preamble signals of IEEE802.11 based OFDM signals and the signal in a time domain is transformed by IFFT at the transmitter. At the receiver, the correlation calculation is employed and period of the short preamble signals is detected. Conventionally, the signal is discarded after the timing synchronization. On the other hand, in the proposed method, the received signals are transformed in the frequency domain by the FFT processing. The interference can be detected by observing the subcarriers which are not mapped short preamble signal using the received signal after the FFT processing. By a computer simulation, it is shown that the propose method can successfully detect interference from the other system. Moreover, we propose the use of orthogonal polarized antennas at the AP and its coupling characteristic is evaluated.

\section{Proposed method}

Fig. 1 (a) shows the frame format when applying the proposed method. This format is used in IEEE802.11n based WLAN system. Fig. 1 (b) shows the waveform of short preamble at the transmitter and receiver to show the interference detection using short preamble signals. The system configuration by the proposed method is shown in Fig. 1 (c). In the IEEE802.11a/g/n based WLAN system, short and long preamble signals are used the timing synchronization and the estimation of channel state information (CSI) between the 


\begin{tabular}{|c|c|c|}
\hline $\begin{array}{l}\text { Short } \\
\text { preamble }\end{array}$ & $\begin{array}{l}\text { Long } \\
\text { preamble }\end{array}$ & Data \\
\hline & $\begin{array}{l}\text { Long } \\
\text { preamble }\end{array}$ & Data \\
\hline
\end{tabular}

(a) Flame format of W-LAN signals.

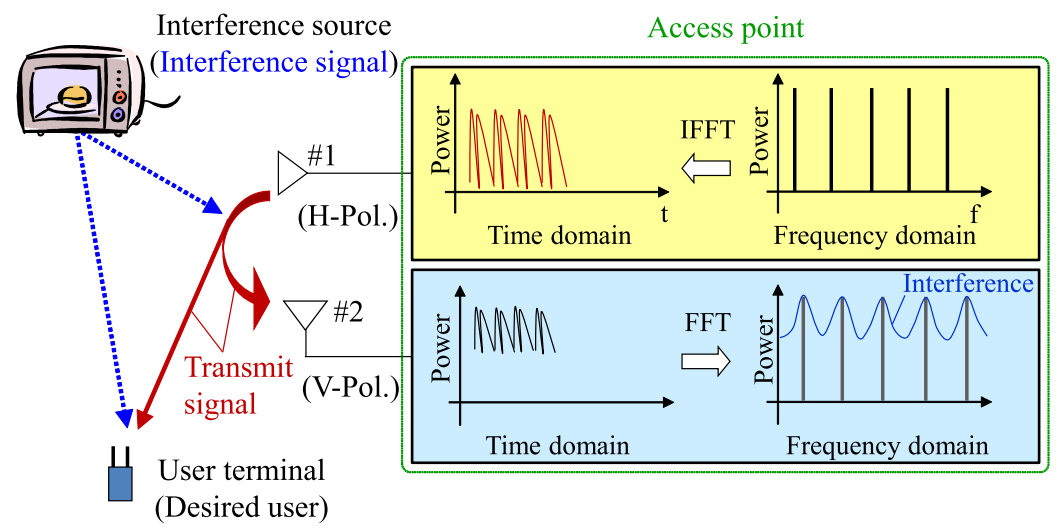

(b) Waveform of short preamble at transmitter and receiver.

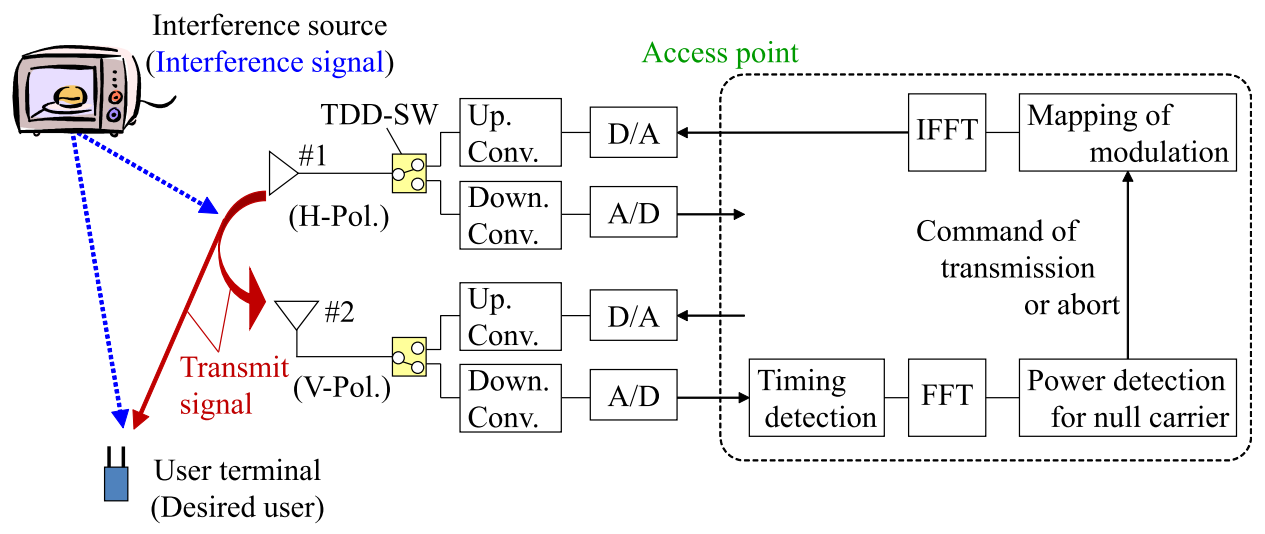

(c) System configuration.

Fig. 1. System configuration by the proposed method.

$\mathrm{AP}$ and UT, respectively.

In the proposed method, we assume $2 \times 2$ MIMO transmission. As can be seen in Figs. 1 (b), the first antenna (Antenna \#1) transmits the short preamble signals to the UT. As shown in Fig. 1 (a), we utilize the fact that the short preamble signals are transmitted by only Antenna \#1. Hence, there is an opportunity for a second antenna (Antenna \#2) to receive the short preamble signals when the transmission by the Antenna \#1 to the UT is employed. When the collision arises, the interference arrives at the Antenna \#2 when the the Antenna \#1 transmits the signals to UT.

Since the power of desired signal from Antenna \#1 to \#2 is much larger than that of interference from IS to AP, the isolation is required between Antenna\#1 and \#2. In this paper, there are following features in the proposed configuration and method.

(A) Isolation between Antennas \#1 and \#2 by using orthogonal polarized antennas. 
(B) Interference detection using received signal after the FFT processing.

As for feature (A), simultaneous transmission (by Antenna \#1) and reception (by Antenna \#2) using orthogonal polarized antennas is proposed. The orthogonal polarized antennas are generally used for reducing the spatial correlation between two antennas and especially effective in outdoor scenario [4], when the MIMO systems are considered. In the proposed method, the orthogonal polarized antennas are used not only the reduction on the spatial correlation but also the isolation for simultaneous transmission and reception. The basic performance in an actual room is evaluated in Section 3.

Regarding the detailed feature of (B), the principle is explained hereafter. Fig. 1 (b) shows the waveform of short preamble at the transmitter and the received signal at the receiver to show interference detection using short preamble signals. As can be seen Fig. 1 (b), the short preamble signal is adopted in IEEE802.11a/n/g based WLAN system. At the transmitter, the signals are mapped for only twelve subcarriers to generate the periodical signal in time domain [1]. The signal is transmitted after the IFFT processing. At the receiver, the timing synchronization between the known transmit and received signals is employed. The correlation value is maximized at the initial timing of the short preamble signals.

In the general WLAN system, the short preamble is not used after the timing synchronization. In this paper, we utilize the short preamble signals after the FFT processing. The received signals after the FFT processing contain the desired signal, interference signal and noise. Interference plus thermal noise can be detected by observing the subcarriers which are not mapped short preamble signal using the received signal after the FFT processing. When the interference power is greater than the noise power, the arrival of interference can be judged by checking the power on the subcarriers which are not mapped short preamble signal. The noise power is used as the threshold value. The noise power can be estimated by power on the subcarriers that the signals are not mapped at the transmitter.

Finally, as can be seen Fig. 1(c), the transmitter stops communicating with UT. Since the proposed method do not need ACK signal unlike CSMA/CA, we confirmed that the transmit efficiency becomes very high and the transmit efficiency by the proposed method is approximately $90 \%$ [3].

\section{Effectiveness of proposed method}

Figs. 2 (a), (b) and (c) shows MIMO-OFDM transceiver and the measurement environment, respectively. IEEE802.11n based MIMO-OFDM signals can be transmitted and received by the transceiver in Figs. 2 (a) and (b) [5]. As shown in Fig. $2(\mathrm{C})$, vertical polarized or orthogonal polarized antennas for the transmit and receive antennas are used for the measurement of mutual coupling. The AP is located at the place in Fig. 2 (c). The center frequency is $2.4 \mathrm{GHz}$. The element spacing is set to be $2.5 \lambda_{0}$. The transmit power is $0 \mathrm{dBm}$ and the average noise power was $-60 \mathrm{dBm}$. 


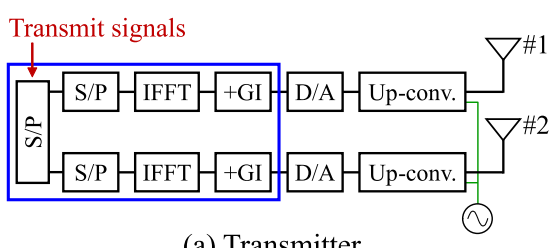

(a) Transmitter.

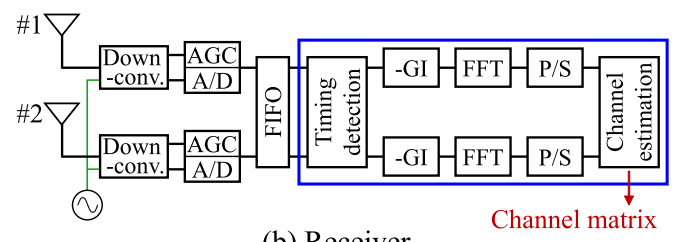

(b) Receiver.

Channel matrix

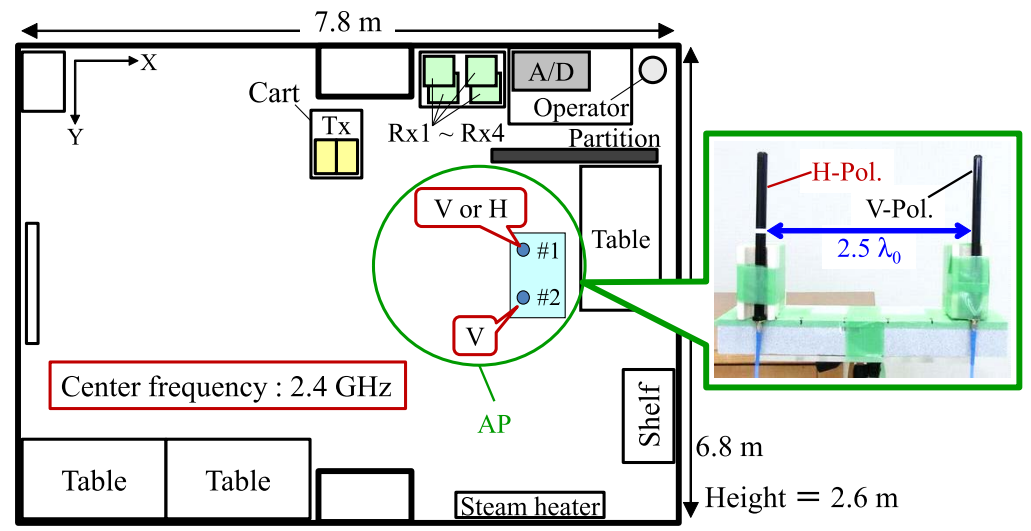

(c) Measurement environment.

Fig. 2. MIMO-OFDM transceiver and measurement environment.

Fig. 3 (a) shows the amplitude of short preamble signal in the frequency [1]. As shown in Fig. 3 (a), only twelve signals are mapped. Fig. 3 (b) shows the measured received power when the short preamble signals are transmitted by actual IEEE802.11n based OFDM signals. The results using only vertical polarized and orthogonal polarized antennas are shown in Fig. 3 (b). As can be seen in Fig. 3 (b), the power reduction is approximately $10 \mathrm{~dB}$ when using vertical polarized antennas due to high mutual coupling. Moreover, the noise power is increased due to the saturation of low noise amplifier at the receiver. On the other hand, $35 \mathrm{~dB}$ power reduction can be observed and noise power is not increased when using orthogonal polarized antennas. Hence, it is shown that the use of orthogonal polarized antennas is effective for the proposed interference detection.

In order to clarify the basic characteristics of the interference detection by the proposed method, the computer simulation using IEEE802.11n based OFDM signals is employed. The signal to interference power ratio (SIR) is set to be 20 and $50 \mathrm{~dB}$. The signal to noise power ratio (SNR) is assumed to be $40 \mathrm{~dB}$. Since the transmit and receive antennas are closely located with each other, the propagation characteristic between the transmit and received antennas is assumed be the AWGN channel. Rayleigh fading is assumed as the propagation channel on the interference by the IS. The other basic parameters are the same with IEEE $802.11 \mathrm{n}$ standard.

Figs. 3 (c) and (d) show the received power in the frequency domain. The solid lines are received powers and the broken lines are the powers of desired signal plus thermal noise. As can be seen Fig. 3 (c), it is possible to judge the interference, because the signals can be detected at the null subcarriers. On the other hand, Fig. 3 (d) indicates that the interference is hidden in the thermal noise. Therefore, it is shown that the interference can be sufficiently 


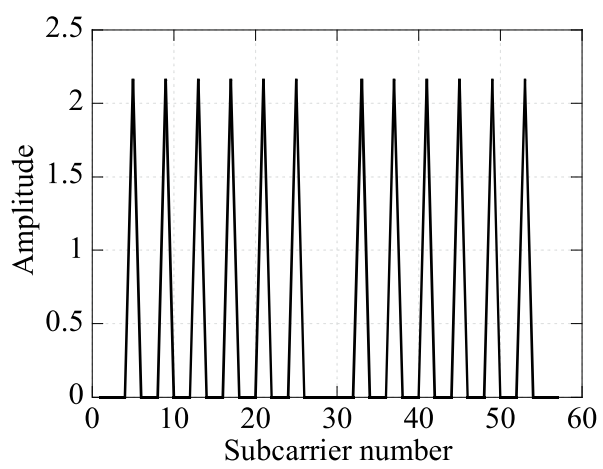

(a) Short preamble.

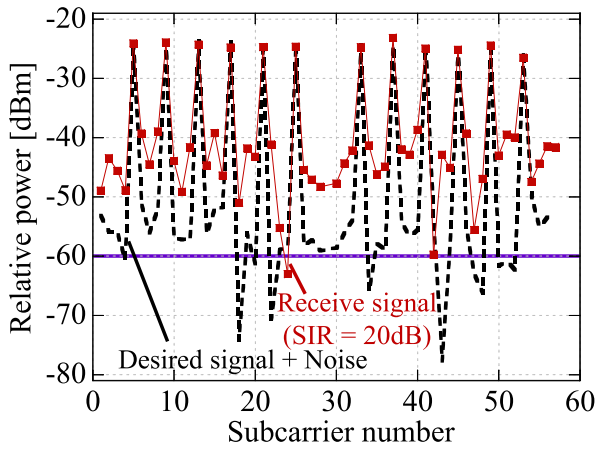

(c) Receive signal $(\mathrm{SIR}=20 \mathrm{~dB})$.

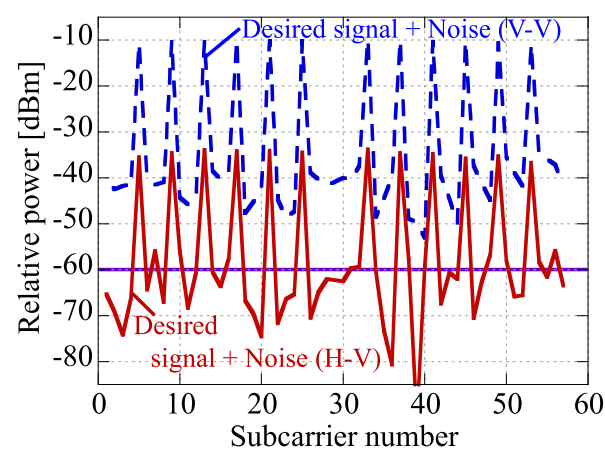

(b) Received power in frequency domain.

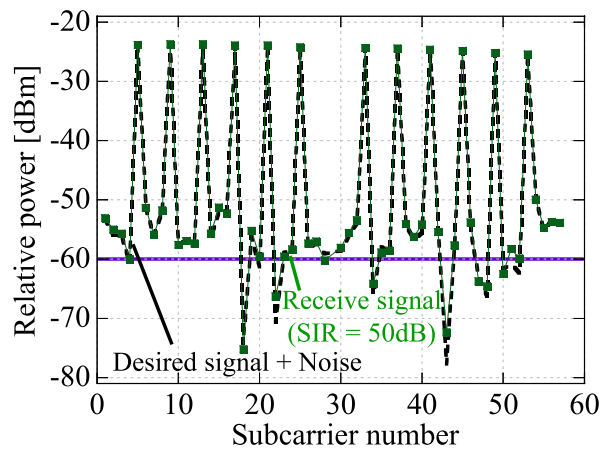

(d) Receive signal $(\mathrm{SIR}=50 \mathrm{~dB})$.

Fig. 3. Received signal of short preamble signal and interference in frequency domain.

detected when the interference power is greater than the noise power. In the future, we will conduct the measurement regarding the interference detection by using the testbed in [5].

\section{Conclusion}

This letter has proposed the interference detection method by utilizing the short preamble signals in the MIMO transmission. The proposed method focuses on IEEE802.11 based short preamble signals which are mapped at only twelve subcarriers in the frequency domain and the simultaneous transmission between transmitter and receiver. By the proposed method, it was shown that the interference from the other system can be detected by observing the null subcarriers which are not mapped the desired signals after the FFT processing. By the measurement using the orthogonal polarized antennas, $35 \mathrm{~dB}$ power reduction between the transmitter and receiver at the $\mathrm{AP}$ was obtained. Moreover, it was found that the interference can be detected when the interference power is greater than the noise power via the computer simulation.

\section{Acknowledgments}

The part of this work was supported by Adaptive and Seamless Technology Transfer Program through Target-driven R\&D (A-STEP), Japan Science and Technology Agency and by the Telecommunications Advancement Foundation. 